\title{
Clinicopathological Significance of Minimal Extrathyroid Extension in Solitary Papillary Thyroid Carcinomas
}

\author{
Chang Gok Woo, MD ${ }^{1}$, Chang Ohk Sung, MD', Yun Mi Choi, $\mathrm{MD}^{2}$, Won Gu Kim, $\mathrm{MD}^{2}$, Tae Yong Kim, $\mathrm{MD}^{2}$, \\ Young Kee Shong, $\mathrm{MD}^{2}$, Won Bae Kim, $\mathrm{MD}^{2}$, Suck Joon Hong, $\mathrm{MD}^{3}$, and Dong Eun Song, $\mathrm{MD}^{1}$ \\ ${ }^{1}$ Department of Pathology, Asan Medical Center, University of Ulsan College of Medicine, Seoul, Korea; ${ }^{2}$ Department of \\ Internal Medicine, Asan Medical Center, University of Ulsan College of Medicine, Seoul, Korea; ${ }^{3}$ Department of Surgery, \\ Asan Medical Center, University of Ulsan College of Medicine, Seoul, Korea
}

\begin{abstract}
Background. The definitive diagnosis of minimal extrathyroid extension (ETE) is subjective because a welldefined true capsule is absent in the thyroid gland. We subclassified the extent of minimal ETE and investigated the clinicopathological significance of the presence of minimal ETE in patients with solitary papillary thyroid carcinomas (PTCs) and solitary papillary thyroid microcarcinomas (PTMCs).
\end{abstract}

Methods. A series of 546 patients with solitary PTCs, including 144 patients with solitary PTMCs, were retrospectively analyzed. Whether the presence of minimal ETE had an effect on recurrence-free survival (RFS) along with other clinicopathological parameters was investigated.

Results. The only independent prognostic factor found to be associated with recurrence was the presence of $\mathrm{LN}$ metastasis in solitary PTC ( $p=0.002)$ but not in solitary PTMC groups ( $p=0.073)$. The presence of minimal ETE had no effect on RFS in both solitary PTC ( $p=0.053)$ and solitary PTMC ( $p=0.816)$.

Conclusions. The presence of minimal ETE has no significant influence on RFS in solitary PTC and PTMC. There is a risk of overrepresenting the $\mathrm{T} 3$ category in solitary PTC and PTMC patients with minimal ETE.

Electronic supplementary material The online version of this article (doi:10.1245/s10434-015-4659-0) contains supplementary material, which is available to authorized users.

(C) The Author(s) 2015. This article is published with open access at Springerlink.com

First Received: 4 February 2015;

Published Online: 16 June 2015

D. E. Song, MD

e-mail: hipuha@hanmail.net
Papillary thyroid carcinoma (PTC) is the most common well-differentiated thyroid carcinoma and is characterized by follicular differentiation and distinct atypical nuclear features. PTC accounts for approximately 85-90\% of differentiated thyroid carcinomas. ${ }^{1}$ Furthermore, the incidental detection of papillary thyroid microcarcinoma (PTMC), with a maximum diameter of $10 \mathrm{~mm}$ or less, has recently increased as a consequence of the frequent use of ultrasonography in regular health checkup. ${ }^{2-5}$

The presence of minimal extrathyroid extension (ETE) in PTCs is defined as when tumor cells extend to the sternothyroid muscle or perithyroid soft tissue. ${ }^{6}$ Most cases of PTCs with minimal ETE exhibit an extension to the perithyroid soft tissue rather than to the sternothyroid muscle. The presence of a minimal ETE is classified as the T3 category in PTCs, irrespective of tumor size, by the American Joint Commission on Cancer (AJCC) cancer staging system (7th edition). ${ }^{6}$ Because clinical recommendations for proper treatment can vary according to the TNM stage, accurate $\mathrm{T}$ categorization is very important. ${ }^{7-9}$ However, histological confirmation of the presence of minimal ETE in PTCs can be both controversial and subjective among endocrine pathologists because of the absence of a well-defined true capsule in the thyroid gland. ${ }^{10-12}$ The thyroid capsule is usually composed of inconspicuous thin fibrous tissues with a variable amount of adipose tissue, blood vessels, and skeletal muscle. It is deficient in the anterior midline of the isthmus, and skeletal muscle can even be mixed with thyroid follicles within the thyroid parenchyma of this area. $^{10-12}$

We classified the extent of minimal ETE and investigated the clinicopathological significance of the presence of minimal ETE to identify any effect on recurrence-free survival (RFS) in patients with solitary PTCs and PTMCs. 


\section{MATERIALS AND METHODS}

\section{Patients}

We retrospectively reviewed 546 patients (484 female, 62 male) with a solitary PTC without evidence of extensive ETE (T4 category). Distant metastases were initially detected according to the current AJCC cancer staging system in patients who underwent total thyroidectomy or hemithyroidectomy, irrespective of cervical lymph node (LN) dissection, at Asan Medical Center, Seoul, Korea, from 1998 to 2003. Patient ages ranged from 8 to 87 years (average 44 years). LN dissection was performed when a LN metastasis was clinically suspicious by ultrasonography (US) and computed tomography (CT) or was histologically confirmed by previous fine needle aspiration biopsy prior to surgery. We reviewed the patient clinical and pathological parameters, including age, gender, procedures, mean tumor diameter, extent of tumor extension, cervical LN metastasis status, and the presence of tumor recurrence (Table 1). This study was approved by the institutional review board of Asan Medical Center.

\section{Pathological Evaluation}

All slides from 546 patients were reviewed by two pathologists, including one experienced endocrine pathologist (D.E.S.). We subclassified the extent of tumor extension into the following three categories: confinement to the thyroid parenchyma (E0), extension to the perithyroid soft tissue (E1), and extension to the sternothyroid muscle (E2). Among 546 patients, $196(35.9 \%)$ were classified as E0, 259 $(47.4 \%)$ were classified as E1, and $91(16.7 \%)$ were classified as E2 (Table 1). Among 546 patients, 334 patients revealed cervical lymph node metastasis at the first operation. We analyzed the extent of the lymph node metastasis at the first operation, which included maximum metastatic tumor size, $\mathrm{N}$ stage, and presence of extranodal extension (Supplementary Table S1).

\section{Follow-up}

All patients with PTCs were regularly followed up every 6-12 months with physical examinations, serum thyroglobulin and anti-thyroglobulin antibody measurements, and US neck examinations. A diagnostic whole body scan was performed after total thyroidectomy and remnant RAI ablation, as previously described. ${ }^{13}$ Following hemithyroidectomy, a CT scan and/or 18F-FDG PET were used to detect recurrence or distant metastasis if clinically suspected. The median follow-up period for patients was 113 (range 1-168) months. Recurrence was defined as structural disease recurrence, such as the reappearance of a
TABLE 1 Demographic and clinicopathologic characteristics of 546 patients with solitary papillary thyroid carcinoma

\begin{tabular}{|c|c|}
\hline Characteristics & Number $(\%)$ \\
\hline \multicolumn{2}{|l|}{ Age (year) } \\
\hline Mean at diagnosis (range) & $44(8-87)$ \\
\hline$<45$ & $275(50.4)$ \\
\hline$\geq 45$ & $271(49.6)$ \\
\hline \multicolumn{2}{|l|}{ Sex } \\
\hline Female & $484(88.6)$ \\
\hline Male & $62(11.4)$ \\
\hline \multicolumn{2}{|l|}{ Procedures } \\
\hline Lobectomy/less than total resection & $95(17.4)$ \\
\hline Total thyroidectomy & $451(82.6)$ \\
\hline No lymph node dissection & $5(0.9)$ \\
\hline Central lymph node dissection & $466(85.3)$ \\
\hline Modified radical lymph node dissection & $75(13.7)$ \\
\hline \multicolumn{2}{|l|}{ Tumor size (mm) } \\
\hline Mean (range) & $20(1-100)$ \\
\hline$\leq 10$ & $144(26.4)$ \\
\hline$>10$ & $402(73.6)$ \\
\hline \multicolumn{2}{|l|}{ Extension } \\
\hline Confinement to thyroid parenchyma & $196(35.9)$ \\
\hline Minimal extrathyroid extension & $350(64.1)$ \\
\hline Perithyroid soft tissue & $259(47.4)$ \\
\hline Sternothyroid muscle & $91(16.7)$ \\
\hline \multicolumn{2}{|l|}{ Cervical lymph node metastasis } \\
\hline Present & $334(61.2)$ \\
\hline Absent & $212(38.8)$ \\
\hline \multicolumn{2}{|l|}{ Recurrence } \\
\hline Present & $61(11.2)$ \\
\hline Median duration (mo, interquartile range) & $39(16-72)$ \\
\hline \multicolumn{2}{|l|}{ Site } \\
\hline Neck level I lymph node & $1(1.6)$ \\
\hline Neck level II, III, IV lymph nodes & $50(81.9)$ \\
\hline Neck level V lymph node & $11(18.0)$ \\
\hline Neck level VI lymph node & $3(4.9)$ \\
\hline Previous resection sites & $12(19.7)$ \\
\hline Lung (distant metastasis) & $4(6.6)$ \\
\hline Inguinal lymph node (distant metastasis) & $1(1.9)$ \\
\hline
\end{tabular}

pathologically confirmed malignant tissue and/or the appearance of a metastatic lesion in other organs by imaging studies during follow-up.

\section{Statistical Analyses}

The Chi squared test and Fisher's exact test for univariate analysis were used along with binary logistic regression and Cox-Hazard regression models for multivariate analysis. To estimate survival rates, survival curves were generated using the Kaplan-Meier method and log- 
rank test, respectively. We used SPSS software version 18.0 and $\mathrm{R}$ statistical software for these analyses. Any $p$ value $<0.05$ was considered to indicate a statistically significant difference.

\section{RESULTS}

\section{Recurrence-Free Survival Outcomes Associated} with the Subclassification of an Extrathyroid Extension

Recurrence (median duration, 39 months; interquartile range, $16-72$ months) occurred in $61(11.2 \%)$ of our patients. Recurrence was identified in diverse sites, including one or more sites and occurred in 3 central cervical LNs (level VI), 62 lateral cervical LNs (level I, II, III, IV, or V), 5 distant metastasis (inguinal LN, 1 case; lung, 4 cases), and 12 previous resection sites (Table 1). Regarding previous resection sites, there were 11 ipsilateral soft tissue (operation bed) after total thyroidectomy, which included 6 clear resection margins and 5 involved margins, and 1 contralateral remnant thyroid parenchyma after lobectomy (Supplementary Table S2).

In the primary PTCs of 61 patients with recurrence, 12 $(6.1 \%)$ were confined to the thyroid parenchyma (E0), $38(14.7 \%)$ extended to the perithyroid soft tissue (E1), and $11(12.1 \%)$ extended to the sternothyroid muscle (E2). There was a more statistically significant difference in the RFS outcomes when two groups (E0 versus $\mathrm{E} 1+\mathrm{E} 2)$ were analyzed than when three groups were analyzed (E0 vs. E1 vs. E2) using the log-rank test with Cox-proportional hazard analysis (Table 2; Supplementary Fig. S1). In another two-group comparison (E0 + E1 vs. E2), the presence of extension to the sternothyroid muscle had no significant effect on RFS. Therefore, we used a two-tier classification scheme of minimal ETE (E0 vs. E1 + E2) based on the AJCC cancer staging system in the subsequent evaluations of the clinicopathological significance of minimal ETE in patients with solitary PTCs.

\section{Clinicopathological Parameters Associated with the Presence of a Minimal ETE}

In 546 patients with solitary PTCs, there was no significant difference in the presence of minimal ETE $(\mathrm{E} 1+\mathrm{E} 2)$ according to gender $(p=0.416)$. Older age (odds ratio [OR] 1.688; $95 \%$ confidence interval [CI] 1.154-2.469; $p=0.007$ ), larger tumor size (OR 3.078; $95 \%$ CI $2.040-4.645 ; p<0.001)$, and the presence of a LN metastasis (OR 2.071; $95 \%$ CI 1.407-3.048; $p<0.001)$ were significantly correlated with the presence of a minimal ETE (Supplementary Table S3).
TABLE 2 Recurrence according to subclassification of invasion in solitary papillary thyroid carcinoma

\begin{tabular}{lllll}
\hline Classification & $\begin{array}{l}\text { Recurrence, } \\
\text { no. }(\%)\end{array}$ & HR & $95 \%$ CI & $p$ value \\
\hline I & & & & \\
E0 & $12(6.1)$ & 1 (ref) & & 0.015 \\
E1 & $38(14.7)$ & 2.444 & $1.277-4.676$ & 0.007 \\
E2 & $11(12.1)$ & 2.076 & $0.916-4.786$ & 0.080 \\
II (current T stage) & & & \\
E0 & $12(6.1)$ & $1($ ref) & & \\
E1 + E2 & $49(14.0)$ & 2.350 & $1.250-4.419$ & 0.006 \\
III & $50(11.0)$ & $1($ ref) & & \\
E0 + E1 & $11(12.1)$ & 1.144 & $0.595-2.198$ & 0.686 \\
E2 & & & & \\
\hline
\end{tabular}

$E O$ confinment to thyroid parenchyma, $E 1$ extension to perithyroid soft tissue, E2 extension to sternothyroid muscle

\section{Clinicopathological Parameters that Affect Recurrence-Free Survival}

In 546 patients with solitary PTCs, females exhibited a lower rate of recurrence (hazard ratio [HR] 0.536; $95 \% \mathrm{CI}$ $0.288-0.997 ; p=0.049$ ). A larger tumor diameter was significantly correlated with a higher recurrence rate $(p=0.019)$ in univariate analysis but not in multivariate analysis (HR 1.616; $95 \%$ CI $0.757-3.451 ; p=0.215$ ). There was a significant difference between types of surgery in univariate analysis $(p=0.020)$ but not in multivariate analysis $(p=0.356)$. The presence of cervical LN metastasis was significantly associated with recurrence (HR $3.173 ; 95 \%$ CI $1.536-6.553 ; p=0.002)$. Among the extent of LN metastasis, $\mathrm{N}$ stage, presence of extranodal extension, and maximum metastatic tumor size were significantly associated with recurrence in univariate analysis, but $\mathrm{N}$ stage had no effect in multivariate analysis (Supplementary Table S4). Additionally, the presence of minimal ETE was significantly correlated with recurrence in univariate analysis $(p=0.008)$; however, there was no significance in multivariate analysis (HR 1.879; $95 \%$ CI 0.992-3.560; $p=0.053$; Table 3). Minimal ETE according to types of surgery had also no effect on recurrence (Supplementary Table S5).

In 144 patients with solitary PTMCs, the presence of minimal ETE was not associated with tumor recurrence in univariate analysis $(p=0.254)$ and multivariate analysis (HR 1.215; $95 \%$ CI 0.235-6.278; $p=0.816$; Table 4). Additionally, there was no significant difference in RFS outcomes between the absence and presence of minimal ETE $(p=0.240$; Fig. 1$)$. The presence of cervical LN metastasis was significantly associated with recurrence in univariate analysis $(p=0.034)$, but there was no 
TABLE 3 Clinicopathologic parameters associated with recurrence of solitary papillary thyroid carcinomas

\begin{tabular}{|c|c|c|c|c|c|c|}
\hline \multirow[t]{2}{*}{ Parameters } & \multirow[t]{2}{*}{ HR } & \multicolumn{2}{|l|}{ Univariate } & \multirow[t]{2}{*}{ HR } & \multicolumn{2}{|l|}{ Multivariate } \\
\hline & & $95 \% \mathrm{CI}$ & $p$ value & & $95 \% \mathrm{CI}$ & $p$ value \\
\hline \multicolumn{7}{|l|}{ Age (year) } \\
\hline$<45$ & 1 (ref) & & & 1 (ref) & & \\
\hline$\geq 45$ & 0.626 & $0.373-1.050$ & 0.076 & 0.670 & $0.397-1.131$ & 0.134 \\
\hline \multicolumn{7}{|l|}{ Sex } \\
\hline Male & 1 (ref) & & & 1 (ref) & & \\
\hline Female & 0.409 & $0.221-0.754$ & 0.004 & 0.536 & $0.288-0.997$ & 0.049 \\
\hline \multicolumn{7}{|l|}{ Procedures } \\
\hline Lobectomy/less than total resection & 1 (ref) & & & 1 (ref) & & \\
\hline Total thyroidectomy & 3.973 & $1.244-12.685$ & 0.020 & 1.798 & $0.517-6.259$ & 0.356 \\
\hline \multicolumn{7}{|l|}{ Size $(\mathrm{mm})$} \\
\hline$\leq 10$ & 1 (ref) & & & 1 (ref) & & \\
\hline$>10$ & 2.348 & $1.159-5.128$ & 0.019 & 1.445 & $0.663-3.151$ & 0.355 \\
\hline \multicolumn{7}{|l|}{ Cervical lymph node } \\
\hline No metastasis & 1 (ref) & & & 1 (ref) & & \\
\hline Metastasis & 3.932 & $1.938-7.978$ & $<0.001$ & 3.173 & $1.536-6.553$ & 0.002 \\
\hline \multicolumn{7}{|l|}{ Invasion } \\
\hline Confinement & 1 (ref) & & & 1 (ref) & & \\
\hline Minimal extrathyroid extension & 2.350 & $1.250-4.419$ & 0.008 & 1.879 & $0.992-3.560$ & 0.053 \\
\hline
\end{tabular}

significant difference in multivariate analysis (HR 7.353; $95 \%$ CI 0.831-65.051; $p=0.073$; Table 4).

\section{DISCUSSION}

The presence of minimal ETE in PTC has been associated with LN metastasis, local recurrence, and poor prognosis. ${ }^{14-16}$ However, recent studies have called into question the prognostic significance of the presence of minimal ETE for tumor recurrence in PTC. ${ }^{17-19}$ Tumors with minimal ETE can be classified as category T3, irrespective of tumor diameter, according to the AJCC TNM cancer staging system. ${ }^{2}$ Thus, most cases of PTMCs can reveal the presence of minimal ETE after microscopic evaluation, especially when tumors are peripherally located despite a small diameter (accounting for $43 \%$ of cases in the present study). Moreover, the benefit of evaluating true capsular invasion to confirm minimal ETE has been debated among endocrine pathologists because the anatomy of the thyroid gland demonstrates no definite fibrous capsule. The adipose tissue and fibers of the skeletal muscle, which are usually observed in the extrathyroid area, are normally also present within the thyroid parenchyma. ${ }^{10,11}$ Although the involvement of skeletal muscle is a more reliable feature for confirming the presence of minimal ETE than involvement of the perithyroid soft tissue, skeletal muscle is not uniformly distributed throughout the thyroid gland and can even be mixed with thyroid follicles in the isthmus. ${ }^{12}$ Identification of thick extrathyroid arteries might be helpful for confirming the presence of minimal ETE, but there is no reliable anatomical landmark that can serve as a criterion for minimal ETE. ${ }^{20}$ In our present study, we microscopically subclassified the extent of minimal ETE to estimate and compare the effect on RFS based on extension to the perithyroid soft tissue or the sternothyroid muscle, respectively. We found that invasion to the sternothyroid muscle had no dominant effect on RFS (Table 2). Furthermore, our current analyses suggested that the current definition for minimal ETE based on the AJCC TNM cancer staging system (E0 vs. E1 + E2) revealed the most statistically significant difference in RFS. ${ }^{2}$

Among the various clinicopathological parameters that we tested, older age, larger tumor size, and the presence of LN metastasis were each found to be associated with the presence of minimal ETE (Table S3), but there was no significant difference in terms of gender. Although male patients exhibited a significantly higher recurrence rate (Table 3) for PTC, these estimates were limited by the relatively small number of male patients $(11.4 \%, 62 / 546)$. Moreover, there was no significant difference in PTMC found to be related to gender. The only independent prognostic factor that we found to be associated with recurrence was the presence of $\mathrm{LN}$ metastasis in our 
TABLE 4 Clinicopathologic parameters associated with recurrence of solitary papillary thyroid microcarcinomas

\begin{tabular}{|c|c|c|c|c|c|c|}
\hline \multirow[t]{2}{*}{ Parameters } & \multirow[t]{2}{*}{ HR } & \multicolumn{2}{|l|}{ Univariate } & \multirow[t]{2}{*}{ HR } & \multicolumn{2}{|l|}{ Multivariate } \\
\hline & & $95 \% \mathrm{CI}$ & $p$ value & & $95 \% \mathrm{CI}$ & $p$ value \\
\hline \multicolumn{7}{|l|}{ Age (year) } \\
\hline$<45$ & 1 (ref) & & & 1 (ref) & & \\
\hline$\geq 45$ & 0.250 & $0.051-1.241$ & 0.090 & 0.324 & $0.062-1.702$ & 0.183 \\
\hline \multicolumn{7}{|l|}{ Sex } \\
\hline Male & 1 (ref) & & & 1 (ref) & & \\
\hline Female & 0.417 & $0.051-3.404$ & 0.414 & 0.905 & $0.108-7.594$ & 0.927 \\
\hline \multicolumn{7}{|l|}{ Procedures } \\
\hline Lobectomy/less than total resection & 1 (ref) & & & 1 (ref) & & \\
\hline Total thyroidectomy & 2.410 & $0.486-11.947$ & 0.281 & 1.277 & $0.213-7.673$ & 0.789 \\
\hline \multicolumn{7}{|l|}{ Cervical lymph node } \\
\hline No metastasis & 1 (ref) & & & 1 (ref) & & \\
\hline Metastasis & 9.599 & $1.181-78.042$ & 0.034 & 7.353 & $0.831-65.051$ & 0.073 \\
\hline \multicolumn{7}{|l|}{ Invasion } \\
\hline Confinement & 1 (ref) & & & 1 (ref) & & \\
\hline Minimal extrathyroid extension & 2.303 & $0.550-9.643$ & 0.254 & 1.215 & $0.235-6.278$ & 0.816 \\
\hline
\end{tabular}

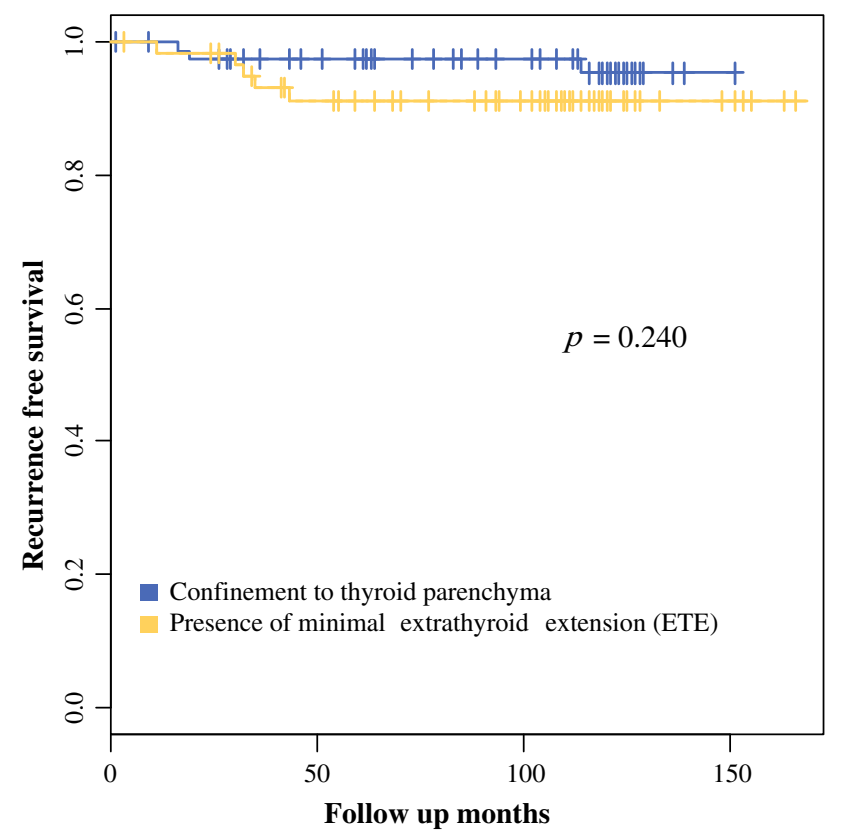

FIG. 1 Recurrence-free survival (RFS) according to presence of minimal ETE in solitary PTMCs. There was no significant difference in the RFS between the absence and presence of minimal ETE in solitary PTMCs $(p=0.240)$

solitary PTC but not in solitary PTMC (Tables 3, 4). In cases with LN metastasis, presence of extranodal extension and macrometastasis was significantly associated with a higher recurrence rate. The reason why there was a significance in $\mathrm{N}$ stage in univariate analysis but no effect in multivariate analysis might be caused by an effect that the presence of extranodal extension and larger metastatic tumor size displayed tendency of higher $\mathrm{N}$ stage. The presence of minimal ETE had no effect on RFS in solitary PTC and PTMC regardless of types of surgery (Tables 3, 4; Fig. 1; Tables S1 and S5). This finding is consistent with the results of previous studies of RFS or relapse-free survival in both PTC and PTMC patient groups. ${ }^{17-19,21}$

Our current retrospective study had several limitations of note, including a small sample size (546 PTCs, 144 PTMCs). A larger study of RFS in patients with PTMCs needs to be performed in the future. Indeed, a larger multicenter study will be needed for the proper validation of our current findings. Various effects of different clinical treatment modalities, such as RAI, were not analyzed for RFS in our present study. We attempted to eliminate the effects of multifocal PTCs in RFS, which limited our analysis to cases with solitary PTCs.

\section{CONCLUSIONS}

The presence of LN metastasis is the only independent prognostic factor associated with RFS in solitary PTC patients. The presence or absence of minimal ETE has no significant effect on RFS in solitary PTC and PTMC. Because the microscopic diagnostic criteria for the presence of minimal ETE remain the subject of debate, there is risk of over assigning patients with PTMC to the T3 category based on the present AJCC TNM cancer staging system. 
OPEN ACCESS This article is distributed under the terms of the Creative Commons Attribution 4.0 International License (http:// creativecommons.org/licenses/by/4.0/), which permits unrestricted use, distribution, and reproduction in any medium, provided you give appropriate credit to the original author(s) and the source, provide a link to the Creative Commons license, and indicate if changes were made.

\section{REFERENCES}

1. Schlumberger MJ. Papillary and follicular thyroid carcinoma. $N$ Engl J Med. 1998;338:297-306.

2. Davies L, Welch HG. Increasing incidence of thyroid cancer in the United States, 1973-2002. JAMA. 2006;295:2164-7.

3. Kim DL, Song KH, Kim SK. High prevalence of carcinoma in ultrasonography-guided fine needle aspiration cytology of thyroid nodules. Endocr J. 2008;55:135-42.

4. De Lellis RA, Lloyd RV HP, Eng C, eds. World Health Organization. Classification of tumours. Pathology and genetics of tumours of endocrine organs. Lyon, France: IARC Press; 2004.

5. Hay ID, Hutchinson ME, Gonzalez-Losada T, et al. Papillary thyroid microcarcinoma: a study of 900 cases observed in a 60year period. Surgery. 2008;144:980-7 (discussion 7-8).

6. Edge SB, Byrd DR. AJCC cancer staging manual, 7th edn. New York: Springer; 2010.

7. Ito $\mathrm{Y}$, Miyauchi $\mathrm{A}$, Inoue $\mathrm{H}$, et al. An observational trial for papillary thyroid microcarcinoma in Japanese patients. World $J$ Surg. 2010; 34: 28-35.

8. Mazzaferri EL. Managing small thyroid cancers. JAMA. 2006;295:2179-82.

9. Pelizzo MR, Boschin IM, Toniato A, et al. Papillary thyroid microcarcinoma (PTMC): prognostic factors, management and outcome in 403 patients. Eur J Surg Oncol. 2006;32:1144-8.

10. Ranade AV, Rai R, Pai MM, et al. Anatomical variations of the thyroid gland: possible surgical implications. Singap. Med J. 2008;49:831-4.

11. SS. Gray's Anatomy, 39th edn. The Anatomical Basis of Clinical Practice. Churchill Livingstone; 2005.
12. Mete O, Rotstein L, Asa SL. Controversies in thyroid pathology: thyroid capsule invasion and extrathyroidal extension. Ann Surg Oncol. 2010;17:386-91.

13. Yim JH, Kim EY, Bae Kim W, et al. Long-term consequence of elevated thyroglobulin in differentiated thyroid cancer. Thyroid 2013;23:58-63.

14. Ortiz S, Rodriguez JM, Soria T, et al. Extrathyroid spread in papillary carcinoma of the thyroid: clinicopathological and prognostic study. Otolaryngol Head Neck Surg. 2001;124:261-5.

15. Arora N, Turbendian HK, Scognamiglio T, et al. Extrathyroidal extension is not all equal: implications of macroscopic versus microscopic extent in papillary thyroid carcinoma. Surgery. 2008;144:942-7. (discussion 7-8).

16. Shaha AR, Shah JP, Loree TR. Risk group stratification and prognostic factors in papillary carcinoma of thyroid. Ann Surg Oncol. 1996;3:534-8.

17. Ito $\mathrm{Y}$, Tomoda $\mathrm{C}$, Uruno $\mathrm{T}$, et al. Minimal extrathyroid extension does not affect the relapse-free survival of patients with papillary thyroid carcinoma measuring $4 \mathrm{~cm}$ or less over the age of 45 years. Surg Today. 2006;36:12-8.

18. Ito $\mathrm{Y}$, Tomoda $\mathrm{C}$, Uruno $\mathrm{T}$, et al. Prognostic significance of extrathyroid extension of papillary thyroid carcinoma: massive but not minimal extension affects the relapse-free survival. World J Surg. 2006;30:780-6.

19. Shin JH, Ha TK, Park HK, et al. Implication of minimal extrathyroidal extension as a prognostic factor in papillary thyroid carcinoma. Int J Surg. 2013;11:944-7.

20. Jeon HM, Lim BJ, Chang HS, Hong S. The definition of minimal extrathyroid extension in thyroid pathology by analyzing sizable intra- and extrathyroid blood vessels. Korean J Pathol. 2012;46: 548-53.

21. Moon HJ, Kim EK, Chung WY, Yoon JH, Kwak JY. Minimal extrathyroidal extension in patients with papillary thyroid microcarcinoma: is it a real prognostic factor? Ann Surg Oncol. 2011;18:1916-23. 